\title{
Using Distributed Wearable Sensors to Measure and Evaluate Human Lower Limb Motions
}

\author{
Sen Qiu, Zhelong Wang, Member, IEEE, Hongyu Zhao, and Huosheng Hu, Senior Member, IEEE
}

\begin{abstract}
This paper presents a wearable sensor approach to motion measurements of human lower limbs, in which subjects perform specified walking trials at self-administered speeds so that their level walking and stair ascent capacity can be effectively evaluated. After an initial sensor alignment with the reduced error, quaternion is used to represent 3-D orientation and an optimized gradient descent algorithm is deployed to calculate the quaternion derivative. Sensors on the shank offer additional information to accurately determine the instances of both swing and stance phases. The Denavit-Hartenberg convention is used to set up the kinematic chains when the foot stays stationary on the ground, producing state constraints to minimize the estimation error of knee position. The reliability of this system, from the measurement point of view, has been validated by means of the results obtained from a commercial motion tracking system, namely, Vicon, on healthy subjects. The step size error and the position estimation accuracy change are studied. The experimental results demonstrated that the extensively existed sensor misplacement and sensor drift problems can be well solved. The proposed self-contained and environment-independent system is capable of providing consistent tracking of human lower limbs without significant drift.
\end{abstract}

Index Terms-Denavit-Hartenberg (DH) convention, foot kinematics, motion tracking, wearable sensors, zero velocity updates (ZUPTs).

\section{INTRODUCTION}

$\mathbf{H}$ UMAN lower limb motion analysis involves qualitative analysis or quantitative evaluation of the human walking, running, and climbing. It is based on kinematic concepts and human anatomy and physiology and widely deployed in medical rehabilitation, augmented virtual reality, foot navigation, and so on. Arnold et al. [1] proposed a lower limb model for human movement analysis based on the muscle architecture and strength parameters. Hornung et al. [2] presented a self-calibrating visual-based system for reconstruction and tracking of arbitrary articulated objects in real time. These existing evaluation methods have

Manuscript received June 14, 2015; revised September 18, 2015; accepted September 20, 2015. Date of publication February 11, 2016; date of current version March 8, 2016. This work was supported in part by the National Natural Science Foundation of China under Grant 61174027 and Grant 61473058 and in part by the Fundamental Research Funds for the Central Universities under Grant DUT15ZD114. The Associate Editor coordinating the review process was Dr. Domenico Grimaldi. (Corresponding author: Zhelong Wang.)

S. Qiu, Z. Wang, and H. Zhao are with the School of Control Science and Engineering, Dalian University of Technology, Dalian 116024, China (e-mail: wangzl@dlut.edu.cn).

H. Hu is with the Department of Computer Science and Electronic Engineering, University of Essex, Colchester CO4 3SQ, U.K.

Color versions of one or more of the figures in this paper are available online at http://ieeexplore.ieee.org.

Digital Object Identifier 10.1109/TIM.2015.2504078 some obvious drawbacks. For instance, electromyographybased methods require dedicated medical apparatus, working space, and professional medical staff. Visual-based methods have the highest accuracy and no drift but require costly optical signal processing equipment. Moreover, a clean background is normally required since any reflective objects can reduce the measurement accuracy.

Due to the advantages of ultrasmall size and low cost, miniature low-power inertial sensors can be mounted directly on the human body for movement monitoring without the need for the specified test environment [3]. In previous studies, Yun et al. [4] and Wang et al. [5] adopted the foot-attached inertial sensor in walking distance calculation. Bebek et al. [6] and Bamberg et al. [7] adopted a pressure sensor array and inertial measurement unit (IMU) for gait measurement. The estimation of arm motion by wearable inertial sensors were demonstrated [8], [9]. The inertial navigation techniques are integrated with active radio-frequency identification (RFID) technology in [10] to locate persons indoors, which obtained accurate results but introduced additional complexity to the system.

There still exist two ubiquitous challenges when wearable sensors are applied to human movement tracking, i.e., initial errors due to sensor misplacement and sensor drift errors, especially in a long period of time. To tackle the problem, Jiang et al. [11] developed a rotation matrix method and obtained good performance when dealing with large sensor misplacement. Similar methods are widely used, but the error correction is less effective when the misplacement is unremarkable.

In this paper, three wearable magnetic angular rate and gravity (MARG) sensors are deployed to calculate instantaneous orientation, velocity, and position of the human lower limbs. To tackle the aforementioned challenges, an initial alignment procedure is conducted to eliminate sensor misdisplacement at the beginning of each trial. A gradient descent method is used to fuse different types of sensor data and zero velocity update (ZUPT) [12] is implemented to cope with sensor drift errors when the foot is at rest on the ground. ZUPT is based on the fact that the foot swings to stance phase periodically during human locomotion and the foot is stationary in stance phase [13]. In this case, the knowledge of when the foot has zero velocity is used to calibrate the accumulated errors since the last ZUPT. Three sensor nodes are separately placed on different parts of lower limbs to form constrained states with the complementary geometric relation, which offer us a way for the error correction of knee 
TABLE I

MARG DATA PERFORMANCE SPECIFICATIONS

\begin{tabular}{cccc}
\hline \hline Unit & Accelerometer & Gyroscope & Magnetometer \\
\hline Dimensions & 3 axes & 3 axes & 3 axes \\
Dynamic Range & $\pm 50 \mathrm{~m} / \mathrm{s}^{2}$ & $\pm 1200 \mathrm{deg} / \mathrm{s}$ & $\pm 750 \mathrm{mGauss}$ \\
Bandwidth (Hz) & 30 & 40 & 10 \\
Linearity (\% of FS) & 0.2 & 0.1 & 0.2 \\
Bias stability (unit $1 \sigma)$ & 0.02 & 1 & 0.1 \\
Alignment Error $(\mathrm{deg})$ & 0.1 & 0.1 & 0.1 \\
\hline \hline
\end{tabular}

position estimation. Finally, the lower limb position and orientation during walking trials could be obtained and validated by the Vicon system (made by Oxford Metrics Limited Company), which is a leading optical motion capture product with a positioning accuracy of $1 \mathrm{~mm}$.

The rest of this paper is organized as follows. Section II describes the system configuration and calibration procedure. Subsequently, we demonstrate the methodology for the lower limb motion analysis in Section III. Then, the experimental results and the algorithm validation by a mark-based Vicon motion tracking system are given in Section IV. Section V presents a discussion about the causes of the estimation error and the criterion of methods selection. Finally, concluding remarks and future research targets are given in Section VI.

\section{System Overview And Calibration}

\section{A. Experimental Scenario and Platform}

In this paper, the research is focused on the estimation of lower limb motions in daily activities such as walking and stair ascent. The MARG sensors are fixed on thigh, shank, and foot, respectively, and the motion estimation results were validated by a Vicon system. An off-the-shelf motion tracking system MTx (made by Xsens Company) is adopted in this research, which consists of a network of wearable sensors. Each wearable sensor has a 3-D accelerometer, a 3-D gyroscope, and a 3-D magnetometer. Table I shows their specifications. Raw sensor data are logged to the digital data box (XBus) placed on the waist at a sampling frequency of $100 \mathrm{~Hz}$ and then transmitted to a host computer via Bluetooth. The wearable sensors are fitted with a stretchable hook-and-loop fastener for comfortable use.

Fig. 1 shows the sensor placement and the Vicon motion tracking system, in which two of the eight Vicon cameras are visible. The sensor attachment, the Vicon marker model, and its structure are shown in the bottom right of Fig. 1. In this case, we could obtain 3-D reference position information and orientation details of the three attached sensor nodes.

Four subjects (two males and two females) have taken part in a preliminary study in the robot laboratory at Essex. They have a mean age of $30 \pm 5$ years, a mean weight $65 \pm 10 \mathrm{~kg}$, and a mean height of $1.70 \pm 0.12 \mathrm{~m}$. None of them has a history of lower limb pathologic conditions. Experiments were carried out in two scenarios. One is in an indoor structured environment, i.e., the Essex Robot Arena. The subjects were asked to perform level walking trials from

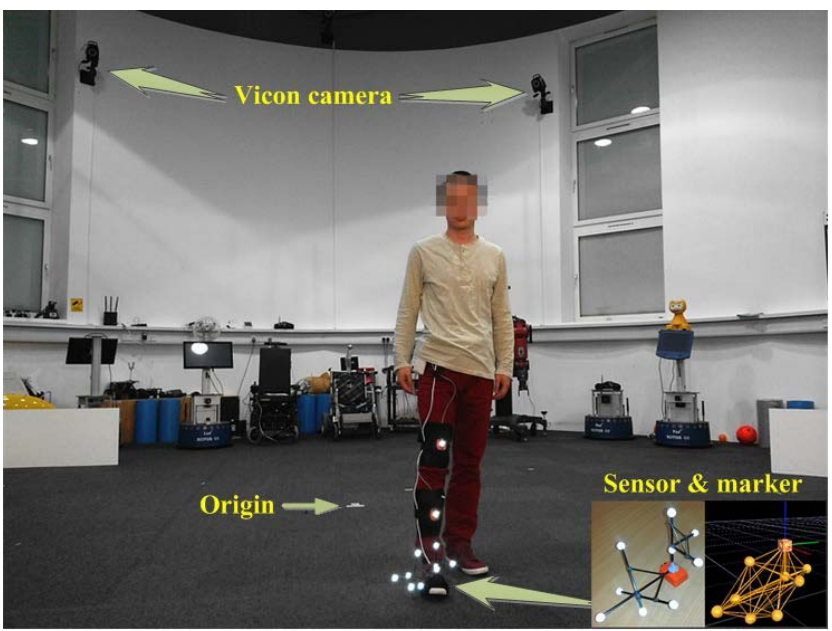

Fig. 1. Overview of the lower limb motion measurement system.

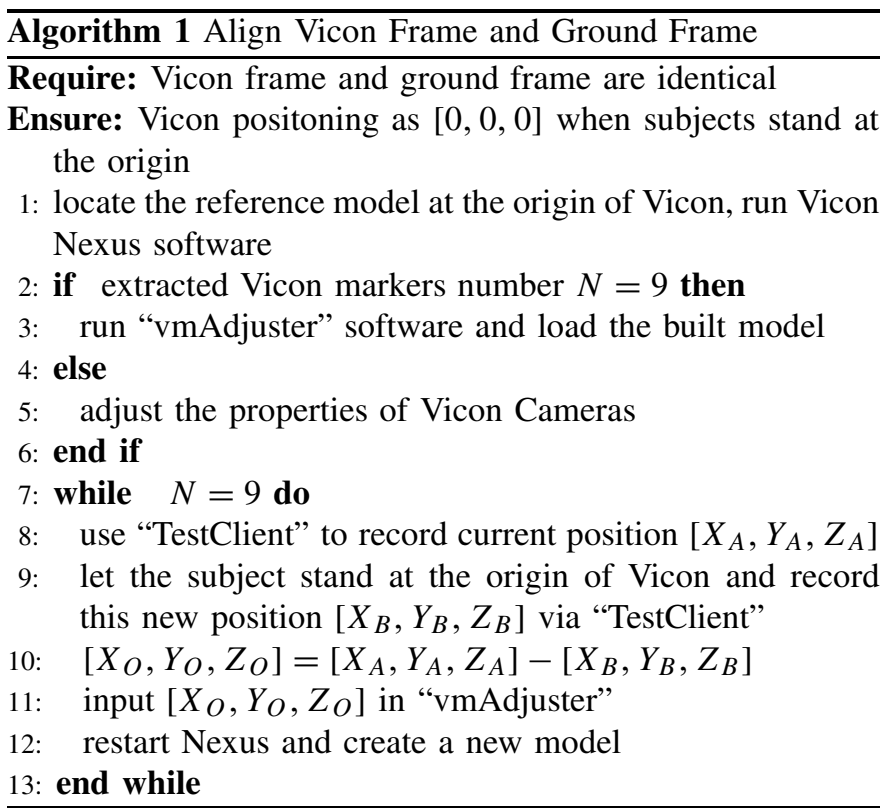

the default origin position and then along the grid lines within the rectangular zone $(5 \times 4 \mathrm{~m})$, which can be effectively monitored by the Vicon system. The second scenario is the staircase in the Network Centre Building. No Vicon is available in this scenario.

There are four coordinate systems involved in our lower limb motion measurement system: 1) the ground frame (G); 2) the MARG sensor frame (S); 3) the body frame (B); and 4) the Vicon frame (V). In operation, we use a reference model with four markers to align $\mathrm{V}$ with $\mathrm{G}$. The pseudocodes of the alignment procedure are presented in Algorithm 1. In this way, Vicon would output the position as $[0,0,0]$ at the beginning of each trial even though the subjects do not start from the specified origin accurately.

\section{B. Initial State Estimation and Sensor Alignment}

Initial orientations were commonly calculated relative to the ground frame defined by the gravity and local magnetic field. 


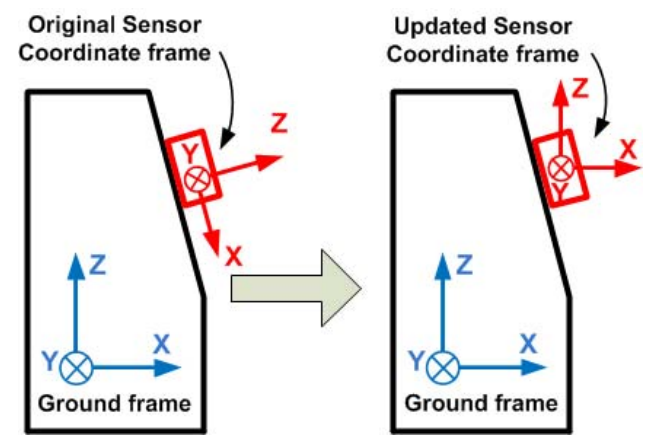

Fig. 2. Initial alignment of the sensor frame.

Noted that the absolute leveled initial position is not common in practical applications, the determination of initial yaw angle requires implicitly the knowledge of the initial roll and pitch angle, which are commonly done by sensing the gravity vector at rest using accelerometers.

We can estimate the initial roll angle $\phi_{\text {ini }}^{\text {roll }}$, pitch angle $\theta_{\text {ini }}^{\text {pitch }}$, and yaw angle $\psi_{\mathrm{ini}}^{\text {yaw }}$, as shown in

$$
\left\{\begin{array}{c}
\phi_{\text {ini }}^{\text {roll }}=\arctan \left(a_{y}, a_{z}\right) \\
\theta_{\text {ini }}^{\text {pitch }}=\arctan \left(-a_{x}, a_{y} * s \phi_{n}^{\text {roll }}+a_{z} * c \phi_{n}^{\text {roll }}\right) \\
\psi_{\text {ini }}^{\text {yaw }}=\arctan \left(m_{y} * c \phi_{n}^{\text {roll }}+m_{x} * s \phi_{n}^{\text {roll }} * s \theta_{n}^{\text {pitch }}\right. \\
\quad-m_{z} * s \phi_{n}^{\text {roll }} * c \theta_{n}^{\text {pitch }}, m_{x} * c \theta_{n}^{\text {pitch }} \\
\left.\quad-m_{z} * s \theta_{n}^{\text {pitch }}\right)
\end{array}\right.
$$

where $c$ and $s$ denote cosine and sine functions, respectively. $a_{x}, a_{y}$, and $a_{z}$ represent accelerometer measurements, and $m_{x}$, $m_{y}$, and $m_{z}$ are magnetometer measurements.

It should be noted that magnetometers are susceptible to local disturbances in the earth magnetic field caused by nearby magnetic objects, which occurs occasionally in routine use. It is necessary to constantly compute the attitude of the sensor in order to correct the measured magnetic values, and a scheme proposed by Fang et al. [14] was adopted in this paper. As discussed above, in order to deal with sensor misplacement, an alignment method similar to bore sighting was used, which is shown in Fig. 2. The implementation is to calculate the initial rotation matrix $R_{\text {trans }}$, which performs the initial transformation of the sensor frame

$$
\begin{aligned}
R_{\text {trans }} & =R_{\psi} R_{\theta} R_{\phi} \\
& =\left[\begin{array}{ccc}
c \psi & -s \psi & 0 \\
s \psi & c \psi & 0 \\
0 & 0 & 1
\end{array}\right]\left[\begin{array}{ccc}
c \theta & 0 & s \theta \\
0 & 1 & 0 \\
-s \theta & 0 & c \theta
\end{array}\right]\left[\begin{array}{ccc}
1 & 0 & 0 \\
0 & c \phi & -s \phi \\
0 & s \phi & c \phi
\end{array}\right] \\
& =\left[\begin{array}{ccc}
c \theta c \psi & s \phi s \theta c \psi-c \phi s \psi & c \phi s \theta c \psi+s \phi s \psi \\
c \theta s \psi & s \phi s \theta s \psi+c \phi c \psi & c \phi s \theta s \psi-s \phi c \psi \\
-s \theta & s \phi c \theta & c \phi c \theta
\end{array}\right] .
\end{aligned}
$$

As defined here, $R_{\text {trans }}$ updates the initial sensor frame system to ensure that all three sensor frames are in alignment. After the initial alignment, the new $x$-axis in the ground frame is chosen by the forward direction of the subject, while keeping the $z$-axis pointing upward. $y$-axis can be obtained according to the rule of the right-handed Cartesian coordinate system as

$$
Y=-(X \times Z) /\|((X \times Z))\| .
$$

\section{Methodology}

\section{A. Orientation Optimization by the Gradient Descent Algorithm}

Quaternion representation is deployed in this research to describe the 3-D orientations of lower limbs. Quaternion requires less calculation time than rotation matrices and is not subject to the existed singularity problem associated with an Euler angle representation. The quaternion is represented as follows:

$$
q=\left(q_{0}, q_{1} i, q_{2} j, q_{3} k\right)
$$

where $i, j$, and $k$ denote the standard orthonormal basis for 3-D space according to the right-handed Cartesian coordinate system. $q_{0}$ is the scalar part of the quaternion, which represents the rotation angle; and $q_{1}, q_{2}$, and $q_{3}$ are the vector part that describes the rotation axis in the 3-D space.

There are no singularity and only two trigonometric functions involved. In this paper, leading superscripts and subscripts are used to identify which coordinate system a quantity is written in. For instance, $q_{S}^{G}$ rotates a vector $x_{S}$ in the sensor frame (S) to $x_{G}$ in the ground reference coordinate system $(G)$

$$
x_{G}=q_{S}^{G} x_{S} q_{S}^{G^{-}}
$$

where $q_{S}^{G^{-}}$is the complex conjugate of $q_{S}^{G}$.

The orientation of a rigid body in space is determined when the axis orientation of a coordinate frame attached to the rigid body is specified with respect to the ground frame. The rigid body angular motion obeys the vector differential equation

$$
\Delta q=\left[\frac{\omega}{\|\omega\|} \sin \frac{\|\omega\| \tau}{2}, \cos \frac{\|\omega\| \tau}{2}\right]^{T}
$$

with regard to the discrete-time system, and we can draw

$$
q(t)=\hat{q}(t-1) \otimes \Delta q
$$

where quaternion product notation $\otimes$ is used to define compound orientations; the ${ }^{\wedge}$ accent denotes a normalized vector with unit length; $\tau$ represents the sample interval $(0.01 \mathrm{~s}$ in this paper); and $\omega_{x}, \omega_{y}$, and $\omega_{z}$ are functions of time, which describe the angular velocities of the three axes.

In terms of physical significance, the orientation change of a rotating frame can be regarded as a rotation about a specific axis with unit vector and scaled by the speed of rotation. In this way, the relationship between the angular rate and quaternion is established. There are many sensor fusion algorithms; the most popular ones are Kalman filters [15], complementary filters [16], and particle filters [17]. Researchers have been committed to the development of simpler methods, which reduce the computational load and parameter tuning burdens associated with the aforementioned algorithms. This part is related to the previous research to make alternative methods of the frequently used extended Kalman filter in applications that require inertial sensing of human motion [18].

The quaternion representation requires a unique $\hat{q}_{S}^{G}$ to be found. This can be achieved by solving an optimization problem. The sensor orientation $\hat{q}_{S}^{G}$ is determined by transforming 
the initial direction of the field in the ground frame $\hat{p}^{G}$ to the sensor frame $\hat{p}^{S}$

$$
\mathbf{f}\left(\hat{q}_{S}^{G}, \hat{p}^{G}, \hat{p}^{S}\right)=\hat{q}_{S}^{G *} \otimes \hat{p}^{G} \otimes \hat{q}_{S}^{G}-\hat{p}^{S} \rightarrow 0 .
$$

Formula (9) calculates the error direction on the solution surface defined by the objective function (8) and the corresponding Jacobian $\mathbf{J}$. While formula (10) presents the gradient descent algorithm for $n$ iterations, which obtains an orientation estimation of $\hat{q}_{S}^{G}$ deduced from the initial orientation $\hat{q}_{S}^{G}(0)$ and a step size $\zeta$. Each new orientation and corresponding sensor measurement are accessible by multiple iterations of formula (10)

$$
\begin{array}{r}
\nabla \mathbf{f}\left(\hat{q}_{S}^{G}, \hat{p}^{G}, \hat{p}^{S}\right)=\mathbf{J}^{T}\left(\hat{q}_{S}^{G}, \hat{p}^{G}\right) \mathbf{f}\left(\hat{q}_{S}^{G}, \hat{p}^{G}, \hat{p}^{S}\right) \\
q_{S}^{G}(t)=\hat{q}_{S}^{G}(t-1)-\zeta \frac{\nabla \mathbf{f}\left(\hat{q}_{S}^{G}, \hat{p}^{G}, \hat{p}^{S}\right)}{\| \begin{array}{l}
\nabla \mathbf{f}\left(\hat{q}_{S}^{G}, \hat{p}^{G}, \hat{p}^{S}\right) \| \\
t=0,1,2, \ldots, n .
\end{array}}
\end{array}
$$

\section{B. Position Estimation With Continuous-Time Sensor Model}

The outputs of calibrated 3-D linear acceleration, rate of turn, and magnetic field data are in the sensor frame (S). It is common to project the acceleration signal $\mathbf{a}_{S}(t)=\left(a_{S} x, a_{S} y, a_{S} z\right)$ obtained from $\mathrm{S}$ into a single optimal estimate of orientation, i.e., the ground frame of reference $(\mathrm{G})$. The physical sensor model is listed in

$$
\left\{\begin{array}{l}
\mathbf{a}_{S}=k_{a} q_{G}^{S}\left(\mathbf{a}_{G}+\mathbf{g}\right) q_{G}^{S^{-}}+B_{a}+N_{a} \\
\boldsymbol{\omega}_{S}=k_{\omega} q_{G}^{S} \boldsymbol{\omega}_{G} q_{G}^{S^{-}}+B_{\omega}+N_{\omega} \\
\mathbf{m}_{S}=k_{m} q_{G}^{S} \mathbf{m}_{G} q_{G}^{S^{-}}+B_{m}+N_{m}
\end{array}\right.
$$

where $a_{S}$ represents the acceleration vector in the sensor frame, $\omega_{S}$ represents the angular velocity vector in the sensor frame, $m_{S}$ represents the magnetic field intensity vector in the sensor frame, and similarly, $a_{G}, \omega_{G}$ and $m_{G}$ are corresponding vectors expressed in ground frame. $\mathbf{g}$ represents the gravity vector; $k_{a}, k_{\omega}$, and $k_{m}$ are the scale factor matrices; $B_{a}, B_{\omega}$, and $B_{m}$ are the bias vectors; and $N_{a}, N_{\omega}$, and $N_{m}$ are uncorrelated white Gaussian measurement noise with zero mean.

In order to obtain accurate data, each sensor node must be properly calibrated to determine sensor nulls and scale factors. The three physical sensors inside each MTx wearable sensor are all calibrated according to physical model of the response of the sensors to various physical quantities, including temperature and misalignment. The basic model is linear and according to

$$
p=K_{W}^{-1}\left(v-b_{T}\right)
$$

where $K_{W}$ represents the weight matrix as shown in (13), $v$ represents the sampled digital voltage, and $b_{T}$ represents the bias vector.

Since the MTx offers the unsigned integer digital voltage outputs from a 16-b $\mathrm{A} / \mathrm{D}$, the calibration data are used to relate the sampled digital voltages $v$ from the sensors to the respective physical quantity $p$

$$
K_{W}=\left[\begin{array}{ccc}
W_{1} & 0 & 0 \\
0 & W_{2} & 0 \\
0 & 0 & W_{3}
\end{array}\right]\left[\begin{array}{lll}
m_{1, x} & m_{1, y} & m_{1, z} \\
m_{2, y} & m_{2, y} & m_{2, y} \\
m_{3, z} & m_{3, z} & m_{3, z}
\end{array}\right]+o
$$

where $W_{n}$ represents the weight coefficient of each axis and $m_{n}$ represents the misalignment, which specifies the direction of the sensitive axes with respect to the ribs of the sensor-fixed coordinate system (S) shell. The three sensitive directions are used to form the misalignment matrix. And $o$ represents higher order models and temperature modeling used for thermal data correction when the environmental temperature changes. In this paper, the cross-axis sensitivity and cross coupling are not taken into account.

The 3-D position and orientation can be calculated by integrating the accelerometer and gyroscope output, respectively, from initial state determined by the accelerometer and magnetometer. The orientation measurements are made in reference to the ground frame of roll, pitch, and yaw (heading). The integration errors of gyroscope tend to grow unbounded because of time-varying biases and wideband measurement noise superimposed onto the gyroscope output [19]. On the other hand, the accelerometer provides drift-free inclination estimates by sensing the gravity field and eliminates drift by continuous correction of the orientation obtained using gyroscope data.

The accelerometers measure all accelerations, including the acceleration due to gravity. The gravity component $\left(\mathbf{g}=[0,0,9.81]^{T} \mathrm{~m} / \mathrm{s}^{2}\right)$ is naturally subtracted from the acceleration signal if one wishes to estimate the displacement by accelerometer. The remaining acceleration is integrated once to calculate liner velocity and twice to obtain displacement. The calculative process is shown in

$$
\left\{\begin{array}{l}
\mathbf{a}_{G}(t)=q_{S}^{G}(t)\left[\mathbf{a}_{S}(t)-\mathbf{g}\right] q_{S}^{G-}(t) \\
\mathbf{v}_{G}(t)=\mathbf{v}_{G}(0)+\int_{0}^{t} \mathbf{a}_{G}(t) d t \\
\mathbf{d}_{G}(t)=\mathbf{d}_{G}(0)+\int_{0}^{t} \mathbf{v}_{G}(t) d t
\end{array}\right.
$$

where $\mathbf{v}_{G}(0)$ and $\mathbf{d}_{G}(0)$ represent the initial liner velocity and the displacement of the subject (both set to zero in this paper), respectively.

Considering that we can only obtain discrete samples from wearable sensors, actually linear velocity and displacement are calculated in the discrete-time domain as shown in

$$
\left\{\begin{array}{l}
\mathbf{v}_{G}(t)=\mathbf{v}_{G}(t-\tau)+\frac{1}{2}\left[\mathbf{a}_{G}(t-\tau)+\mathbf{a}_{G}(t)\right] * \tau \\
\mathbf{d}_{G}(t)=\mathbf{d}_{G}(t-\tau)+\frac{1}{2}\left[\mathbf{v}_{G}(t-\tau)+\mathbf{v}_{G}(t)\right] * \tau .
\end{array}\right.
$$

The validity of above formulas is subject to the assumption that the change of angular velocity is linear in the interval $[t-\tau, t]$.

\section{Segmentation of Gait Cycle}

Previously researchers have defined a gait cycle as a period of time between any two identical kinematic events in the 


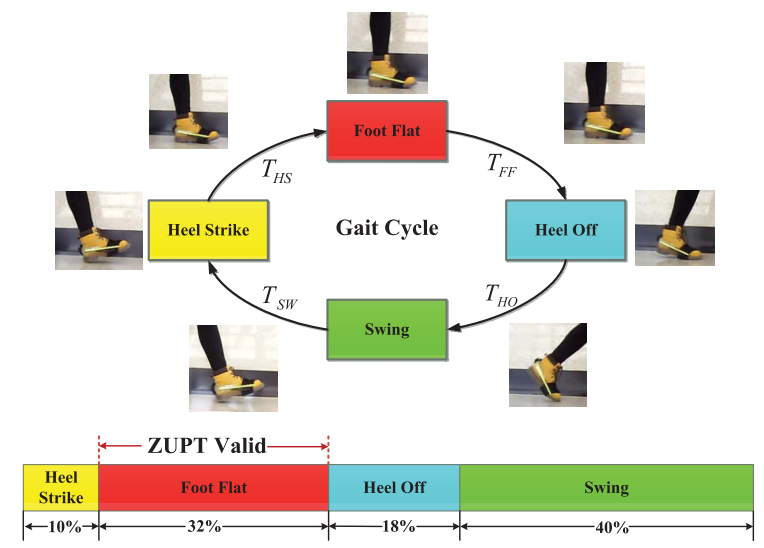

Fig. 3. Typical gait phase in a gait cycle.

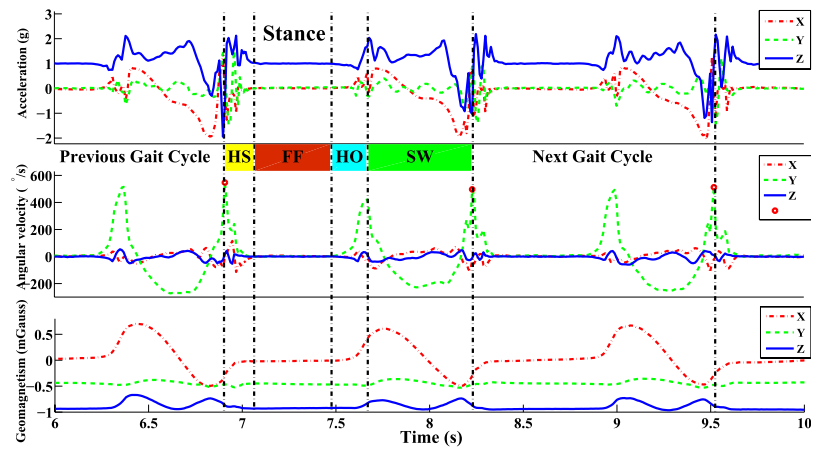

Fig. 4. Segmentation of gait phase using sensor data.

walking cycle, and any event could be selected as the onset of a gait cycle [20]. The heel strike (HS) is commonly selected as the beginning of a stride. In general, there are two ways of dividing a gait cycle.

1) The gait cycle is divided into four different phases in some research work, namely, HS, foot flat (FF), heel off (HO), and swing (SW).

2) In other research, each gait cycle is divided into two phases, i.e., the stance phase when the foot is on the ground and the SW phase when the same foot is in the air [4], [21]. In this division, the stance phase begins with HS and ends with $\mathrm{HO}$, constituting roughly $60 \%$ of the gait cycle, while the SW phase constituting the remaining $40 \%$.

Since our MARG system provides both linear and angular velocities of lower limbs of a subject, we adopt four phases in this paper, as shown in Fig. 3. Note that each individual has a unique gait pattern, and thus the percentage of gait cycle that corresponds to each phase slightly varies between literature sources. The ZUPT interval is deemed sufficient to correct the error growth in the motion estimation step by step.

The gait cycle is characterized by the following features, as shown in Fig. 4, in which three gait cycles are observed.

1) The HS phase starts with a peak of angular velocity follow the SW phase of the previous gait cycle and is accompanied by a spike of acceleration, which contributes to define the boundary between successive strides.

2) The FF phase is the significant phase during which angular velocity becomes near zero and the acceleration

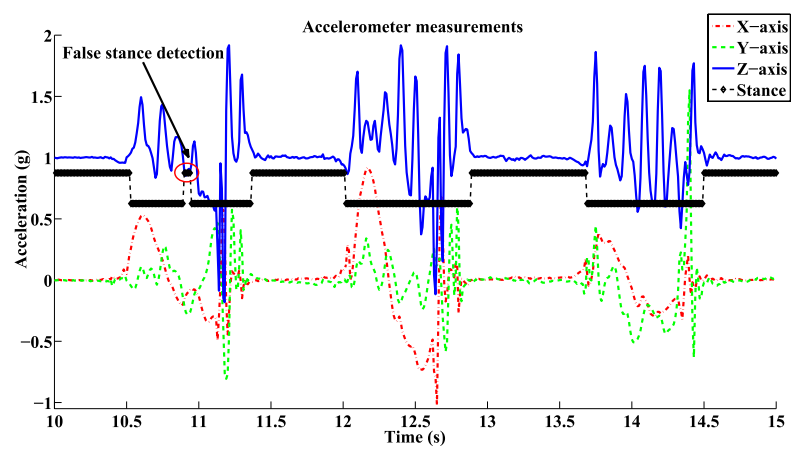

(a)

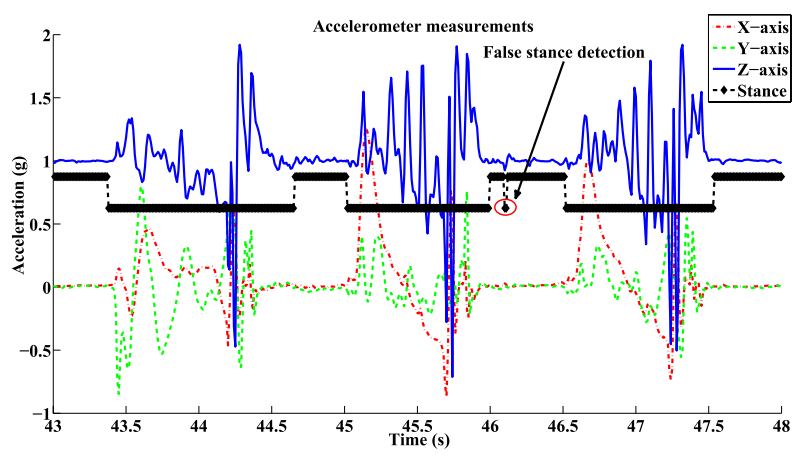

(b)

Fig. 5. False stance detection using a single threshold. (a) False stance detection during the SW phase. (b) False SW detection during the stance phase.

value remains essentially constant with one unit of gravitational acceleration. This is the phase during which ZUPT can be applied.

3) The HO phase begins with the end of the adjacent FF and ends with a peak of angular velocity; meanwhile, another spike of acceleration is presented.

4) The SW phase features a spacing between two peaks of angular velocity and ends with the start of the following HS phase, and the acceleration changes sharply during this phase.

Compared with the acceleration measurements, the angular velocity measurements by a gyroscope provide more prominent features for gait phase segmentation. In this case, the gait cycle can be divided into the aforementioned four phases by the key peaks of angular velocity in conjunction with the criteria of ZUPT, which are detailed as follows.

The periodicity of walking allowing us to repeat this cycle step by step without accumulating significant errors. To ensure the effectiveness of ZUPT, it is critical to identify exactly when the user's foot is at rest on the ground. In [21], stance phase detection methods normally rely on the magnitude or shortterm statistics of acceleration, angular rate, or the combination of two factors, and compare these to predefined thresholds to identify the stance phase. In practical terms, there are a number of small measurement values in short term during the SW phase, and the detection method may detect false stance phase when the foot is actually in the air, as shown in Fig. 5(a). On the other hand, since there are a number of measurement fluctuations during the stance phase, the detection method may interrupt the true stance phase by the 
false SW phase when the foot is actually on the ground, as shown in Fig. 5(b). Thus, to avoid incorrect detection of the stance phase, Yun et al. [4] introduced a sample count $\gamma$ to filter out momentary dips and sudden spikes in the gyroscope measurement. The parameter was incremented until a specified number of samples had satisfied the minimum count condition. This approach is useful, but has its limitation. It assumes a roughly consistent gait pattern during walking. However, the subject may change his/her pace significantly, or even come to a halt in random walk. Therefore, it is not practical to select the optimal minimum count number.

In this paper, two criterions are deployed to distinguish stance phase accurately, then the foot position measurement is updated each step using ZUPT under the assumption that when the subject stands steady on the ground (usually $0.2 \sim 0.4 \mathrm{~s}$ ), the foot attached sensor should be presumed to be in the stationary state. In order to prevent the current drift error propagating to the next step, we calculate the stationary state by taking the squared Euclidean norm of acceleration

$$
A=\sqrt{\left(\operatorname{acc}_{x} /\|\mathbf{g}\|\right)^{2}+\left(\operatorname{acc}_{y} /\|\mathbf{g}\|\right)^{2}+\left(\operatorname{acc}_{z} /\|\mathbf{g}\|\right)^{2}}
$$

where $\operatorname{acc}_{x}, \operatorname{acc}_{y}$, and $\mathrm{acc}_{z}$ represent the triaxial acceleration measurement.

Due to the measurements of accelerometers are constant under stationary state and the magnitude of composed acceleration vector is equal to local gravity, the acceleration moving variance is denoted by the first criterion

$$
\operatorname{Var}_{A}^{2}(j)=\frac{1}{M} \sum_{i=j-M}^{i=j}\left(\left(A_{i}-\bar{A}_{M}\right)^{2}<\lambda_{\text {acc }}\right.
$$

where $\bar{A}_{M}$ is the mean of $A_{i}$ over $M$ samples and $\lambda_{\text {acc }}$ is the empirically predefined threshold.

Meanwhile, angular rate energy $E^{\omega}$ is adopted as a second criterion (18). The second origin moment rather than second central moment is used to detect the stance phase. The detection method provides a preferable alternative form of the statistics estimator, which is defined in the following energy detector [12]:

$$
E_{j}^{\omega}=\frac{1}{\sigma_{\omega}^{2} N} \sum_{i=j}^{j+N-1}\left\|\omega_{i}\right\|^{2}<\lambda_{\text {gyro }}
$$

where $N$ is the window size selected according to the sensor's sampling rate, $\omega_{i}=\left[\omega_{x, i}, \omega_{y, i}, \omega_{z, i}\right]^{T}$ is the triaxial angular velocity vector at the instant of data acquisition time, and $\sigma_{\omega}^{2}$ is the gyroscope noise variance. $\lambda_{\text {gyro }}$ is the predefined threshold that is configured to the same value as $\lambda_{\text {acc }}$. We continually find the interval when ZUPT is valid and update the corresponding $\mathbf{v}_{G}(t)$ as $[0,0,0]^{T}$ base on the above two criterions. In general, the stance phases are first detected by formula (17) and formula (18), and then the rest gait phases are automatically determined by gyroscope data, which have distinct peak values in every single gait cycle.

\section{Forward Kinematic Model}

It should be noted that ZUPT method is valid only when zero velocity assumption is satisfied. Therefore, ZUPT is invalid for knee position estimation because there is no static moment for the knee during walking. Likewise, distortion could happen toward the motion tracking of other parts of human body other than feet. The skeleton structure of human lower limbs makes it applicable to set up geometrical constraints [22], which is based on the concept of forward kinematics from the robot domain.

The classic Denavit-Hartenberg (D-H) convention provides an effective way to demonstrate the lower limb kinematics. The sensor on the shank offers additional information to determine the instances of the stance phase, which is based on the assumption that the foot must be in the stance phase when the accelerometer on the shank senses the minimum forward acceleration after HS. And at this time, the shank is assumed to be vertical to the ground. Since human lower limbs can be treated as a model of inverted pendulum and the geopotential energy reaches the maximum at the minimum of kinetic energy (the vertical moment), which is the start time of the implementation of D-H convention.

Knee position estimation is evaluated by combining the accurate position information of the foot and the D-H convention. Lower limbs are divided into three links, i.e., thigh, shank and foot. Whatever motion the limb executes, the coordinates of each point on link $i$ are constant when expressed in the $i$ th coordinate frame. Furthermore, when joint $i$ is actuated, link $i$ and its attached frame $o_{i} x_{i} y_{i} z_{i}$ experience a resulting motion. The frame $o_{0} x_{0} y_{0} z_{0}$, which is attached to the limb base (foot in this paper), is referred to as the initial frame.

In this paper, lower limb joints are considered as the revolute joint rather than prismatic owning of the biological feature. In this convention, each homogeneous transformation $A_{i}$ shown in formula (19) is represented as a product of three basic transformations. $A_{i}$ is a function of only a single joint variable, namely, $\theta_{i}$

$$
\begin{aligned}
A= & \operatorname{Rot}_{y, \theta_{i}} \operatorname{Trans}_{x, a_{i}} \operatorname{Rot}_{x, \alpha_{i}} \\
= & {\left[\begin{array}{cccc}
c \theta_{i} & -s \theta_{i} & 0 & 0 \\
s \theta_{i} & c \theta_{i} & 0 & 0 \\
0 & 0 & 1 & 0 \\
0 & 0 & 0 & 1
\end{array}\right]\left[\begin{array}{cccc}
1 & 0 & 0 & L_{i} \\
0 & 1 & 0 & 0 \\
0 & 0 & 1 & 0 \\
0 & 0 & 0 & 1
\end{array}\right] } \\
& \times\left[\begin{array}{cccc}
1 & 0 & 0 & 0 \\
0 & c \alpha_{i} & -s \alpha_{i} & 0 \\
0 & s \alpha_{i} & c \alpha_{i} & 0 \\
0 & 0 & 0 & 1
\end{array}\right] \\
= & {\left[\begin{array}{cccc}
c \theta_{i} & -s \theta_{i} c \alpha_{i} & s \theta_{i} c \alpha_{i} & L_{i} c \theta_{i} \\
s \theta_{i} & c \theta_{i} c \alpha_{i} & -c \theta_{i} s \alpha_{i} & L_{i} s \theta_{i} \\
0 & s \alpha_{i} & c \alpha_{i} & 0 \\
0 & 0 & 0 & 1
\end{array}\right] }
\end{aligned}
$$

where $c$ and $s$ denote cosine and sine functions, respectively. The three quantities associated with link $i$ and joint $i$, namely, $\theta_{i}, \alpha_{i}$, and $L_{i}$, are generally joint angle, link twist, and link length, respectively.

It is by nature that $L_{i}$ is constant for a given link, and $\theta_{i}$ and $\alpha_{i}$ for a revolute joint are the variables. While the matrix $A_{i}$ for the knee joint is a function of a single variable $\theta_{i}$ because there are $2 \mathrm{DOF}$ at the knee joint, the knee joint can be 


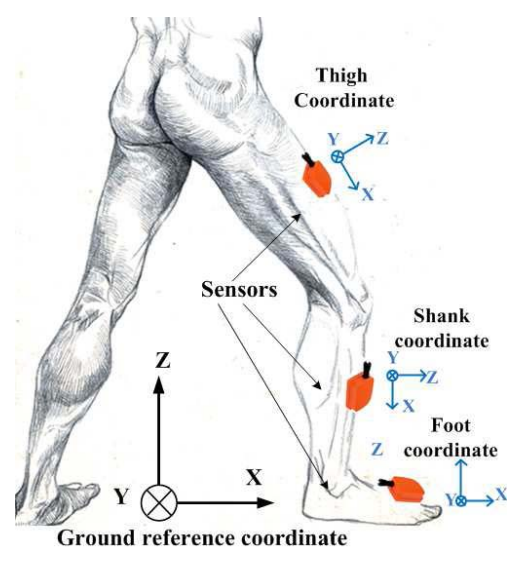

(a)

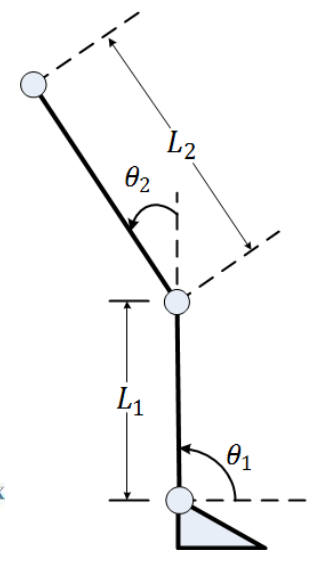

(b)
Fig. 6. Sagittal plane sketch of the human lower limbs. (a) Sensor attachment. (b) D-H model.

assumed as a modified bicondylar hinge joint with only 1 DOF when the according foot is under the stance phase.

Fig. 6 shows the profile pattern of the human foot, shank, and thigh. A conventional link-segment model is used to represent the anatomical human lower limbs. Each segment is represented by a rigid body segment with segmental information of linear acceleration and angular velocity. The linksegment model characterizes physiological joints as hinge points. $L_{1}$ is the distance between the centers of the ankle joint and the knee joint. $L_{2}$ is the distance between the knee joint and the hip joint. It can be measured using an anthropometry, i.e., a device designed to measure the dimensions of the human body.

The forward kinematics assign the Cartesian position and orientation of the sensor frame attached to the human lower limbs relative to the ground reference frame. The mathematic model is given by multiplying a series of matrices parameterized by joint angles, where $\theta_{1}$ and $\theta_{2}$ are rotating angles in local frames originated at the hip and knee joints, respectively, with the initial ankle joint angle $\theta_{1}$ equal to $90^{\circ}$ and the joint angle $\theta_{2}$ with the calculated value by the MARG sensor at the shank vertical moment. The coordinate frames and their transformation are shown in Fig. 6(a) to associate different frames.

Since there are three joints to be located in this study, three MTx sensors are placed on the thigh, shank, and foot, respectively. Based on the $\mathrm{D}-\mathrm{H}$ convention, we can propose link transformations from the ankle joint to the hip joint. This is based on the fact that we can use ZUPT method [12] to prevent error from accumulating step by step. The footattached sensor is relatively more authentic than the estimation from sensors on the shank and thigh. Position correction can be performed during each stance period.
Suppose that all the sensors were kept still before the sampling starts and the displacement of each sensor is relevant to its original position. Let $\left[x_{h}^{G}, y_{h}^{G}, z_{h}^{G}\right]^{T}$ be the displacement vector of the hip joint computed from the angular variations of the thigh, $\left[x_{k}^{G}, y_{k}^{G}, z_{k}^{G}\right]^{T}$ the displacement vector of the knee joint based on the rotation of the shank, and $\left[x_{a}^{G}, y_{a}^{G}, z_{a}^{G}\right]^{T}$ the displacement vector of the ankle joint using the accelerations from the sensor placed on the foot. We then have two constraint states

$$
\begin{aligned}
& \int_{0}^{t} \int_{0}^{t} a_{k}^{G} d t=\left[x_{k}^{G}, y_{k}^{G}, z_{k}^{G}\right]^{T}+\left[x_{h}^{G}, y_{h}^{G}, z_{h}^{G}\right]^{T} \\
& \int_{0}^{t} \int_{0}^{t} a_{a}^{G} d t=\int_{0}^{t} \int_{0}^{t} a_{k}^{G} d t+\left[x_{a}^{G}, y_{a}^{G}, z_{a}^{G}\right]^{T} .
\end{aligned}
$$

In this case, we need to integrate the angular rate near the knee joint so as to locate the knee joint. Suppose that $\varphi, \phi$, and $\psi$ are Euler angles around the $x-, y$-, and $z$-axis, corresponding to the ankle. Then, we have (22), as shown at the bottom of this page, where $c$ and $s$ denote cosine and sine functions, respectively.

In this way, we could substantially reduce drifts in $\left[x_{k}^{G}, y_{k}^{G}, z_{k}^{G}\right]^{T}$ estimation using the constraint equations (20) and (21). If necessary, $\left[x_{h}^{G}, y_{h}^{G}, z_{h}^{G}\right]^{T}$ position can be obtained using a similar method. The flowchart of the proposed approach is shown in Fig. 7.

\section{EXPERIMENTAL RESULTS AND Algorithm VALIDATION}

\section{A. System Calibration and Initialization}

After installation of the sensors, the subjects were instructed to stand still for $5 \mathrm{~s}$ in order to estimate and make adjustments for the tilt of the sensors by measuring the acceleration due to gravity and the geomagnetic field. The relation among the three fixed frames, i.e., thigh $(\mathrm{T})$, shank $(\mathrm{S})$, and foot $(\mathrm{F})$, can be defined during this period. Then the proposed initial alignment procedure guaranteed that all three sensors share the same initial state. In order to test the uniformity among multiple sensors, a uniformity test was carried out. The subject was requested to perform three actions in succession: 1) lift leg to the right; 2) kick forward; and 3) externally rotate the foot. Each action repeated twice followed by the next one.

During this process, lower limbs should be kept straight to ensure that different sensors sense the same rate of change at the same time. Gyroscope data from thigh- and shank-attached sensors were used to calculate roll, pitch, and yaw, as shown in Fig. 8(a). After initial alignment, the blue curve (thigh) is almost coincided with that of the red curve (shank). The difference between three axes is shown in Fig. 8(b). The corresponding errors in the three axes between sensors attached

$$
\left\{\begin{aligned}
x_{k}^{G} & =\frac{L_{1} \pm L_{1} \sqrt{1-\left[1+(c \varphi c \phi c \psi)^{2}\right]\left[1-c^{2} \psi+c^{2} \phi c^{2} \psi-(c \varphi c \phi c \psi)^{2}\right]}}{1+(c \varphi c \phi c \psi)^{2}} \\
y_{k}^{G} & =\sqrt{L_{1}^{2}-z_{k}^{G}} s \psi \\
z_{k}^{G} & =\sqrt{L_{1}^{2}-x_{k}^{G}} s \varphi
\end{aligned}\right.
$$




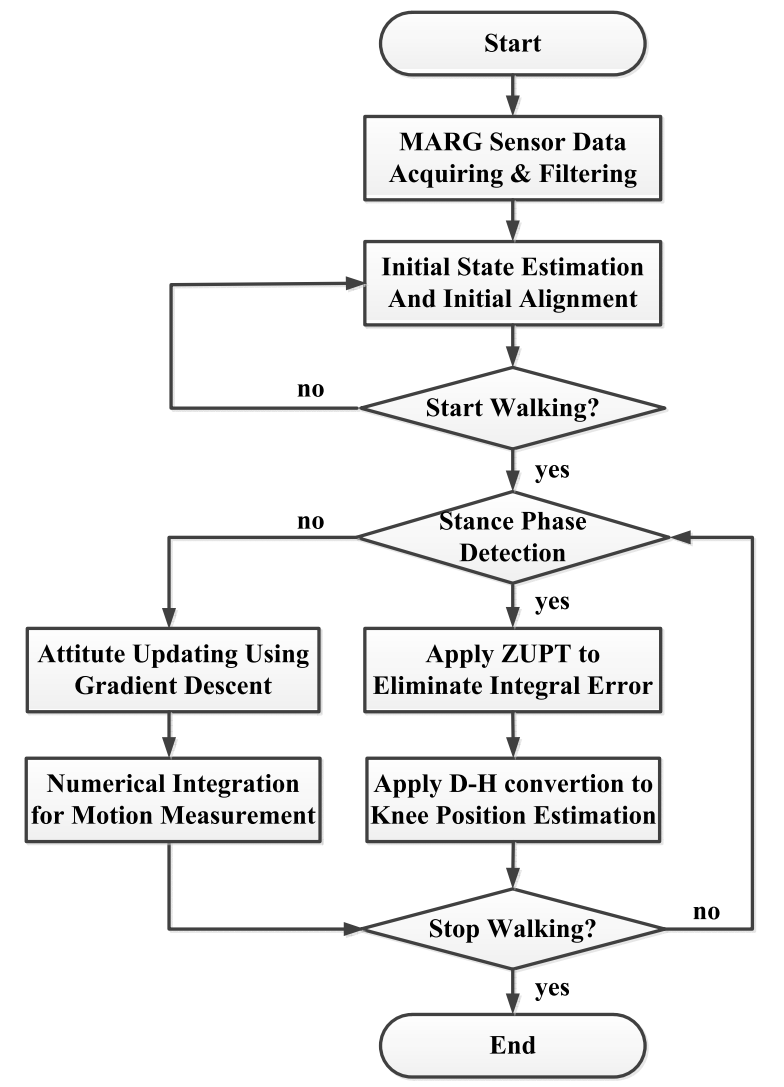

Fig. 7. Flowchart of the motion measurement system.

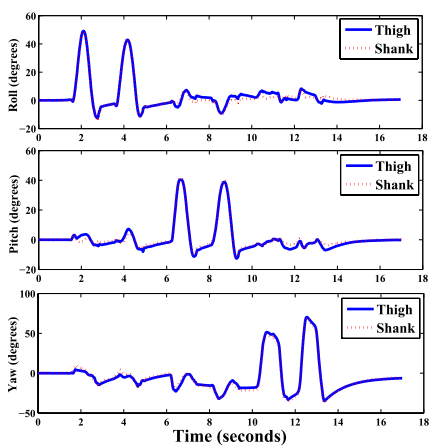

(a)

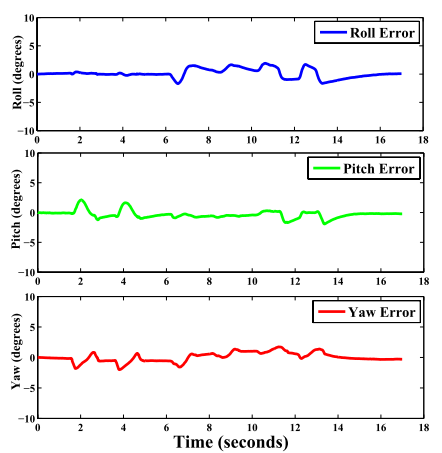

(b)
Fig. 8. Alignment test of multiple sensors. (a) Orientation variation with respect to three test actions. (b) Orientation computational error between two sensors.

to the thigh and the shank reduce to less than $2^{\circ}$, establishing the base of subsequent computations.

Similarly, in order to ensure that the measurements of orientation and position in the Vicon camera coordinate frame are comparable with the estimation algorithm in the ground frame, an initial calibration procedure was performed where a five-marker mini calibration frame was used. This step is required as the cameras may shift over time. When the calibration was done, each marker can be seen by at least six cameras, which is enough for the system evaluation.

\section{B. Orientation and Position Estimation}

Table II shows the elements of the quaternion for the first six samples. The practical position of the sensors after the
TABLE II

QUATERNION CONVERGENCE

\begin{tabular}{ccccc}
\hline \hline Sample & $q_{0}$ & $q_{1}$ & $q_{2}$ & $q_{3}$ \\
\hline 1 & 0.99989 & 0.00246 & 0.00428 & 0.01157 \\
2 & 0.99997 & 0.00195 & 0.00335 & 0.01237 \\
3 & 0.99996 & 0.00199 & 0.00296 & 0.00296 \\
4 & 0.99998 & 0.00181 & 0.00319 & 0.01156 \\
5 & 0.99998 & 0.00178 & 0.00247 & 0.01097 \\
6 & 0.99998 & 0.00172 & 0.00172 & 0.01095 \\
\hline \hline
\end{tabular}

TABLE III

Gait Phase Segmentation

\begin{tabular}{ccccc}
\hline \hline Sujects & $\begin{array}{c}\text { Female } \\
1.58 \mathrm{~m}, 43 \mathrm{~kg}\end{array}$ & $\begin{array}{c}\text { Female } \\
1.66 \mathrm{~m}, 54 \mathrm{~kg}\end{array}$ & $\begin{array}{c}\text { Male } \\
1.76 \mathrm{~m}, 74 \mathrm{~kg}\end{array}$ & $\begin{array}{c}\text { Male } \\
1.83 \mathrm{~m}, 86 \mathrm{~kg}\end{array}$ \\
\hline $\mathrm{SL}(\mathrm{m})$ & $1.054 \pm 0.057$ & $1.132 \pm 0.117$ & $1.213 \pm 0.034$ & $1.306 \pm 0.161$ \\
$\mathrm{ST}(\mathrm{s})$ & $1.189 \pm 0.094$ & $1.209 \pm 0.034$ & $1.281 \pm 0.108$ & $1.232 \pm 0.096$ \\
$\mathrm{HS}(\mathrm{s})$ & $0.131 \pm 0.129$ & $0.097 \pm 0.081$ & $0.150 \pm 0.015$ & $0.119 \pm 0.016$ \\
$\mathrm{FF}(\mathrm{s})$ & $0.404 \pm 0.045$ & $0.406 \pm 0.030$ & $0.375 \pm 0.043$ & $0.386 \pm 0.149$ \\
$\mathrm{HO}(\mathrm{s})$ & $0.190 \pm 0.062$ & $0.218 \pm 0.053$ & $0.282 \pm 0.039$ & $0.239 \pm 0.033$ \\
$\mathrm{SW}(\mathrm{s})$ & $0.464 \pm 0.097$ & $0.488 \pm 0.038$ & $0.474 \pm 0.054$ & $0.501 \pm 0.041$ \\
\hline \hline
\end{tabular}

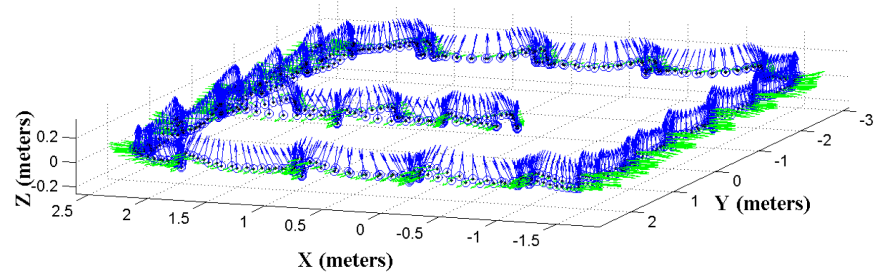

Fig. 9. Foot trajectory of figure-of-eight walking for two laps.

initial alignment is represented by the quaternion $(1,0,0,0)$ in the reference frame. The quaternion estimation converged to the practical position in a couple of iterations.

Note that walking cadence is more stable in straight line walking without turning, which is an advantage for gait cycle segmentation. In this case, four subjects completed the level walking trials along the straight line. The mean value and standard deviation of their gait parameters, including stride length (SL), stride time, and the durations of HS, FF, HO, and $\mathrm{SW}$, are presented in Table III.

The experimental results show that men perform larger steps and longer SL than women. There is a positive correlation between stature and SL. By calculating the percentage of each walking phase in the whole gait cycle, the average values are as follows: HS (10.1\%), FF (32.05\%), HO (18.85\%), and SW $(39.25 \%)$. From the statistical data of the four subjects, the proportion of each gait phase in a gait cycle has been shown to be dependent on walking cadence, which is consistent with [23] and [24].

One of the subject's walking trajectory in the first scenario is shown in Fig. 9, with a profile similar to figure-of-eight. The subject was instructed to walk along the predefined path, from the origin $(x=0, y=0, z=0)$ and initial orientation 


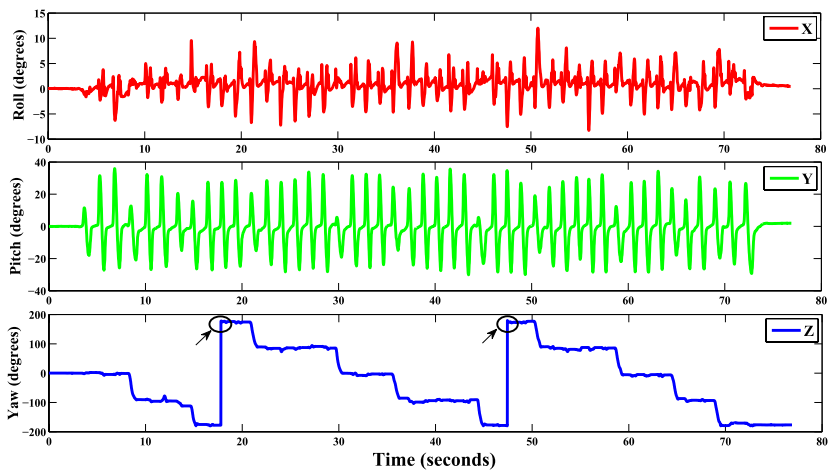

Fig. 10. 3-D foot orientation estimation of figure-of-eight level walking for two laps.

(roll $=0$, pitch $=0$, yaw $=0$ ), which is presented by Vicon. It is hard to maintain the same ending position for different trials. The dominant foot that the sensor was attached to is located at the origin. The subject walked forward ( $x$-axis) from the origin and then along the clockwise direction of the rectangular grid line for two laps, ending up at $(x=-0.125, y=-0.038, z=0.011)$ with a reverse yaw angle $\left(\right.$ roll $=0.43^{\circ}$, pitch $=1.96^{\circ}$, yaw $\left.=-176.69^{\circ}\right)$ with respect to the initial state. The blue arrows in Fig. 9 show the vertical direction ( $z$-axis) of the foot with the attached sensor and the green arrows represent the left direction ( $y$-axis) with respect to the forward direction, which is not shown in the figure in view of legibility.

Similarly, 3-D orientations are shown in Fig. 10. Due to the singularity problem of Euler method, there are some trip points when the yaw is close to $180^{\circ}$, which means the value would jump from $-180^{\circ}$ to $180^{\circ}$. In this experiment, the subject followed the clockwise and turned right nine times, i.e., the yaw angle changed $9 \times\left(-90^{\circ}\right)$ in the ground frame. According to the definition of yaw angle, the value should be in the range $-180^{\circ}$ to $180^{\circ}$. In this case, we adjusted the sign of yaw angle whenever the subject was walking at the reverse direction relative to the initial direction, as shown by black ellipses in Fig. 10, ensuring that the yaw angle always fall in the standard range.

Although the distance error was the main concern in [4], [6], and [25], it is worth researching how the step size error shifts over time in the course of experiments. In this paper, SL is defined as the distance between the adjacent foot landing locations, which is one of the most essential gait parameters that determine the walking speed. With regard to level walking trials, the SL $L$ can be calculated by

$$
L_{i}=\sqrt{\left(x_{i}-x_{i-1}\right)^{2}+\left(y_{i}-y_{i-1}\right)^{2}}
$$

where $L_{i}$ represents the step length of $i$ th step. $x_{i}$ and $y_{i}$ denote the $X$ and $Y$ coordinates in the ground frame, respectively.

Fig. 11 presents the quantitative SL estimation throughout the walking experiment in a rectangular route with 28 detected steps. Each SL is represented by blue bars calculated by the proposed method and green bars given by Vicon, respectively. The step size error is represented by the red bar. The overall

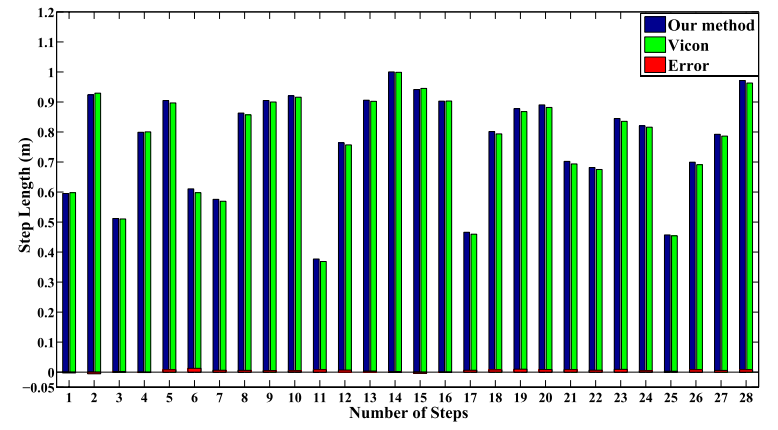

Fig. 11. SL estimation and step size error of walking along the rectangular route for one lap.

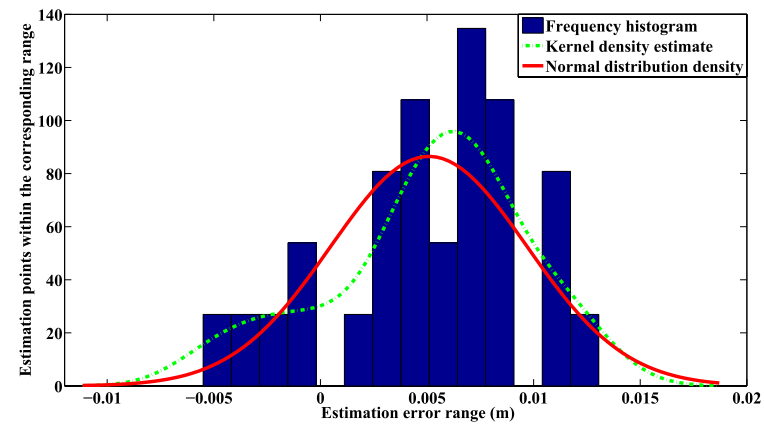

Fig. 12. Error distribution in the form of frequency histogram, kernel density estimation, and normal distribution fitting.

estimation error by our method is slightly larger $(0.41 \%)$ than that of Vicon, which is regarded as ground truth. Specifically, the measured results are less than the real value for five steps and larger than the real value as to the remaining 23 steps. Maximum error appears in the sixth step with $0.006 \mathrm{~m}(1.14 \%)$, and $\mu$ and $\sigma$ are $0.472 \%$ and $0.436 \%$, respectively.

As we can see from the bar graph, step size error does not grow with the increase in step number, though the errors are relatively larger when the subject changes the walking direction, i.e., the steps to make the turning at each corner. To get a more clear understanding of the error distribution, Fig. 12 indicates the corresponding frequency histogram, kernel density estimation, and normal distribution fitting. The corresponding mean and standard deviation are $\mu=0.0051$ and $\sigma=0.0046$. From the statistical analysis, we can draw that the level walking position estimation is effective and the estimation errors are well controlled.

Stair ascent capacity has perioperative prognostic medical importance [26]. It may predict postoperative recovery and complications for stroke rehabilitation. Moreover, the $z$-axis displacement component is more distinct than the level walking trial. The Vicon system does not work in staircase, so we measured the layer height $(0.16 \mathrm{~m})$ and width $(0.3 \mathrm{~m})$. Meanwhile, we marked the origin and ending point with marking pen at each trial. Therefore, we can still obtain ground truth in stair ascent experiments. A subject was asked to climb two flights of stairs, where each flight consisted of ten steps. The trajectory of staircase ascent is shown in Fig. 13 and the 3 -D position ends at $(-0.016,-0.945,3.308)$. It is 


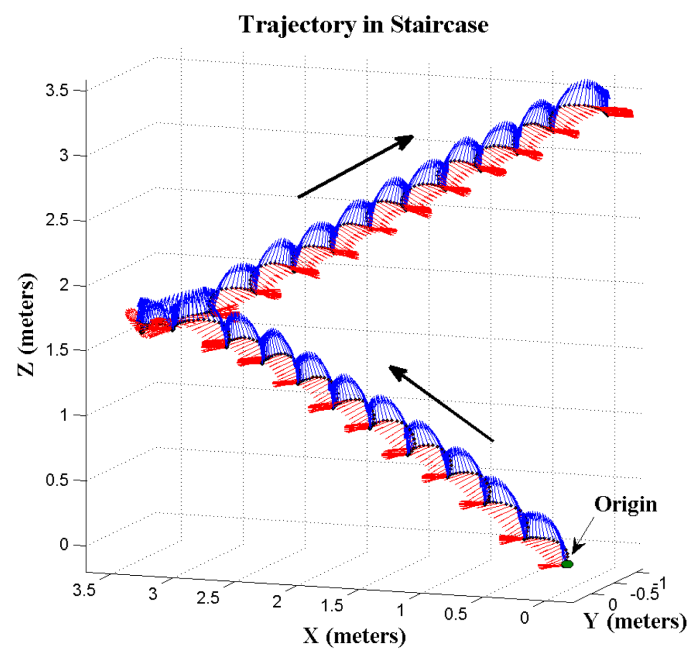

Fig. 13. Foot trajectory of stair ascent.

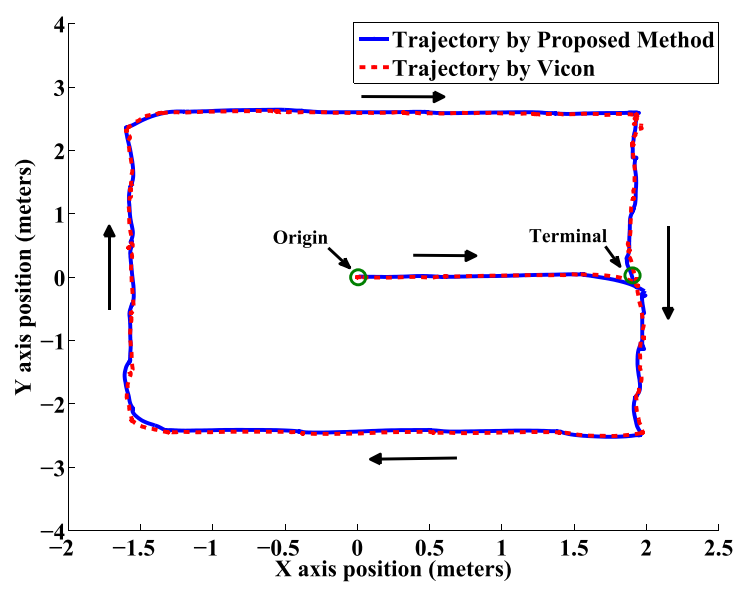

(a)

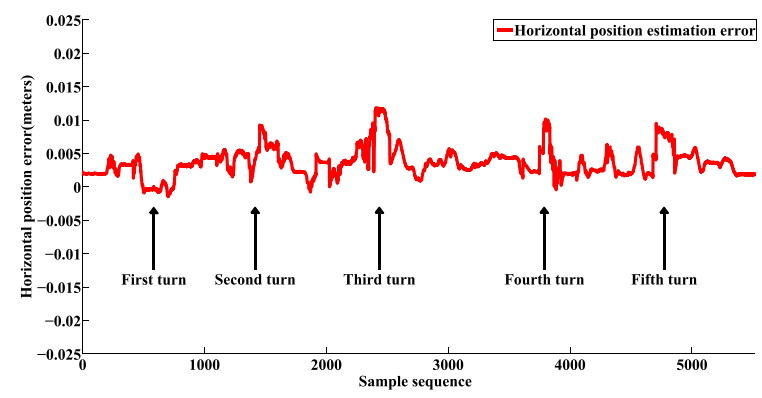

(b)

Fig. 14. Foot trajectory during level walking along the rectangular route (a) Estimation by the proposed method and Vicon, respectively. (b) Horizontal position error $(\triangle X Y)$ between the two results: the calculated value minus the real value.

acceptable compared with the available ground truth. The obtained orientation and position information can be useful for the evaluation of stair ascent capacity.

To verify the effectiveness of the wearable-sensor-based estimation algorithm, a comparison was made between the trajectory estimation by the proposed method and the measurements of Vicon, as shown in Fig. 14. The subject was asked to perform the rectangular route level walking for one

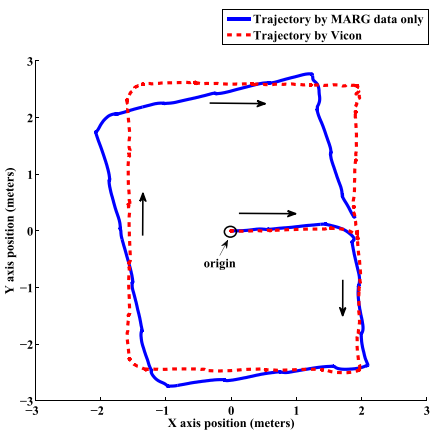

(a)

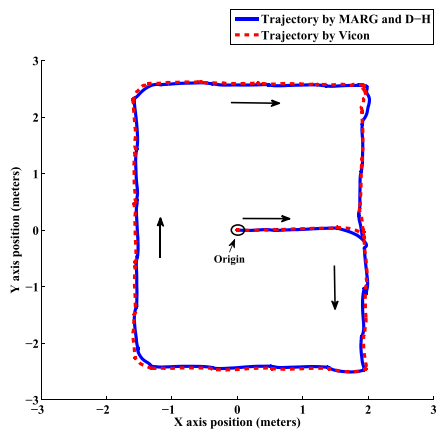

(b)
Fig. 15. Absolute horizontal knee trajectory. (a) Estimation by the MARG algorithm and Vicon, respectively. (b) Estimation by the MARG algorithm combined with D-H convention and Vicon, respectively.

TABLE IV

EXPERIMENTAL PERFormanCE OF POSITION Estimation

\begin{tabular}{ccccc}
\hline \hline Trials & $\begin{array}{c}\text { Step count } \\
\text { (True value) }\end{array}$ & $\begin{array}{c}\text { Distance (m) } \\
\text { (True value) }\end{array}$ & $\begin{array}{c}\text { Distance } \\
\text { \% Error }\end{array}$ & $\begin{array}{c}\text { Step-size } \\
\% \text { Error }\end{array}$ \\
\hline Straight line & $13(13)$ & $15.168(15.164)$ & 0.26 & $0.15 \pm 0.33$ \\
One lap (CW) & $28(28)$ & $21.624(21.591)$ & 0.15 & $0.09 \pm 0.24$ \\
One lap (CCW) & $30(30)$ & $22.241(22.169)$ & 0.32 & $0.21 \pm 0.35$ \\
Two laps & $47(47)$ & $40.736(40.665)$ & 0.17 & $0.18 \pm 0.51$ \\
Four laps & $85(85)$ & $74.139(73.852)$ & 0.39 & $0.30 \pm 0.37$ \\
Stair ascent & $46(46)$ & $6.400(6.353)$ & 0.74 & $0.56 \pm 0.44$ \\
\hline \hline
\end{tabular}

lap in clockwise direction. As shown in Fig. 14(b), there exist some significant errors especially when the subject made the five turnings, which may be due to the signal delay of the wearable sensors. However, the horizontal position estimation error $(\Delta X Y)$ is less than $0.02 \mathrm{~m}$. The comparative results are satisfactory on the whole.

Knee position estimation was evaluated to validate the proposed D-H convention method. In the first case, we implemented the algorithm merely by means of data from the MARG sensor attached to the knee. Obviously, there exist large errors, as shown in Fig. 15, due to the absence of ZUPT. This is because the error in the previous step was introduced to the next step inevitably. Fig. 15(b) shows that the knee trajectory estimation has been greatly improved by the introduction of D-H convention even though there still exist visible errors. In this experiment, the values of $L_{1}$ and $L_{2}$ of the specified subject are 0.43 and $0.52 \mathrm{~m}$, respectively.

Table IV presents six trials that consist of one-straight-line level walking, four-rectangular-route level walking, and onestair ascent walking of two floors. Step numbers, \% error of walking distance, and the step size \% error of SL are summarized. The results indicate that all steps in different trials are detected correctly. Without addition sensors for stance phase detection, the proposed self-contained measurement system achieved an average distance error of $0.26 \%$ for level walking and $0.74 \%$ for stair ascent trials within the indoor environment. What is more, the average step size error is less than $0.2 \%$. It is unconvincing to directly compare the results with those 
of other researches, since the system performance is closely associated with several elements, including system configuration, sensor attachment, and road condition. Our experimental results are comparable or better than some good performance in the literature: the position accuracy of $0.3 \%$ reported in [21], the $\triangle X Y$ radial distance error of $0.82 \%$ and the distance error of $0.27 \%$ reported in [4], and the position error of $0.4 \%$ presented in [25].

\section{DISCUSSION}

It can be concluded from the experimental results that the estimation results by the proposed method are quite similar to the results of the Vicon system on the whole, and the estimation errors are quite small. It means that the proposed method has the ability to track the lower limb motion with high accuracy. Via modeling the human lower limb skeleton structure as a three-link model, it can solve the knee joint position estimation drift problem effectively and correct the drift errors that are summarized as follows.

1) One source of the overall motion estimation errors is due to the wearable sensors attached to nonrigid bodies. In contrast, Vicon measures the movements of the surface of lower limbs where markers are attached, while the D-H convention in our method estimates the movement of the segments of the lower limbs. Therefore, the knee position estimation is worse than the foot position estimation.

2) The other source for the estimation error is the misalignment of coordinate frames. Despite trying our best, the Vicon coordinate frame was not completely aligned with the ground frame, which could introduce estimation difference between these two systems.

In general, wearable sensors suffer obvious drifts after a few minutes of operation. The solution of reducing or correcting drift error for human motion tracking generally fall into three categories: hybrid systems, absolute positioning systems, and specific domain assumptions. Hybrid systems using IMU signals together with signals from additional sensors including force sensor [12], RFID [10], optical sensor [27] or other fore-mentioned sensor system. Some merely use two separate sensors and choose an optimal estimation at a particular time instant [28], and others just apply one method for sensing orientation changes and another for sensing position. With respect to hybrid systems, multidisciplinary fusion remains a big challenge. Absolute positioning systems such as GPS can maintain drift-free position estimation, but unsuitable for indoor applications and high-accuracy applications such as human motion estimation.

In some cases, it is possible to make domain-specific assumptions about the body movement by exploiting constraints, which are confirmable to apply to subject movement tracking. Such assumptions can be adopted to minimize drift effectively. A typical example is ZUPT applied to the situation in which foot-attached sensors are used to track walking trajectory, which is adopted in this paper.

Kinematic chains can be an effective tool when multiple wearable sensors are used to track human limb motions. Due to the physical attributes, human lower limbs can be represented by the rigid body model. If the length of each segment is definite, the D-H convention can be adopted to set up the kinematic chains, which produces state constraints so that the drift error can be minimized. The main drawback of domain specific assumptions is that the assumptions must be valid. Note that D-H convention would not work in stair ascent trials because lower limbs cannot be considered as inverted pendulum when the subject climbs stairs. Therefore, we merely use the data from the foot-attached sensor and just evaluate the foot motion measurement performance accordingly.

\section{CONCLUSION}

The combination of distributed wearable sensors with the D-H convention proposed in this paper resulted in a promising tool for tracking lower limb movements. The experimental results have been presented to show that drift errors were well controlled by the sensor fusion algorithm. The proposed method has been evaluated with the experimental data collected from multiple subjects at self-selected walking speeds. The method developed for lower limb motion measurement in this paper has potential applications for augmented and virtual reality, rehabilitation, emergency responders, and so on. In conclusion, the main contributions of this paper are as follows.

1) The algorithm presented in this paper employs processes similar to others but combine the information of wearable sensors, domain-specific assumptions, and the kinematic chain.

2) Ensure the successful implementation of the ZUPT algorithm and expand the scope of the ZUPT algorithm to the lower limbs other than the foot.

3) The footstep of each stride can be correctly detected, thus avoiding significant drift and providing more satisfactory positioning accuracy for different experimental scenarios.

However, due to the limitation of experimental scene and limited subjects, the experiments are not sufficient to set up a gait analysis database. The estimation method is offline, so it is not feasible to give real-time feedback. Future work is underway to address these problems and further evaluate both healthy and pathological subjects at a wider movement range.

\section{REFERENCES}

[1] E. M. Arnold, S. R. Ward, R. L. Lieber, and S. L. Delp, "A model of the lower limb for analysis of human movement," Ann. Biomed. Eng., vol. 38, no. 2, pp. 269-279, Feb. 2010.

[2] A. Hornung, S. Sar-Dessai, and L. Kobbelt, "Self-calibrating optical motion tracking for articulated bodies," in Proc. IEEE Int. Conf. VR, 2005, pp. 75-82.

[3] J. M. Lambrecht and R. F. Kirsch, "Miniature low-power inertial sensors: Promising technology for implantable motion capture systems," IEEE Trans. Neural Syst. Rehabil. Eng., vol. 22, no. 6, pp. 1138-1147, Nov. 2014.

[4] X. Yun, J. Calusdian, E. R. Bachmann, and R. B. McGhee, "Estimation of human foot motion during normal walking using inertial and magnetic sensor measurements," IEEE Trans. Instrum. Meas., vol. 61, no. 7, pp. 2059-2072, Jul. 2012.

[5] Z. Wang, S. Qiu, Z. Cao, and M. Jiang, "Quantitative assessment of dual gait analysis based on inertial sensors with body sensor network," Sensor Rev., vol. 33, no. 1, pp. 48-56, 2013. 
[6] O. Bebek et al., "Personal navigation via high-resolution gait-corrected inertial measurement units," IEEE Trans. Instrum. Meas., vol. 59, no. 11, pp. 3018-3027, Nov. 2010.

[7] S. J. M. Bamberg, A. Y. Benbasat, D. M. Scarborough, D. E. Krebs, and J. A. Paradiso, "Gait analysis using a shoe-integrated wireless sensor system," IEEE Trans. Inf. Technol. Biomed., vol. 12, no. 4, pp. 413-423, Jul. 2008.

[8] H. J. Luinge, P. H. Veltink, and C. T. M. Baten, "Ambulatory measurement of arm orientation," J. Biomech., vol. 40, no. 1, pp. 78-85, Jan. 2007.

[9] H. Zhou and H. Hu, "Reducing drifts in the inertial measurements of wrist and elbow positions," IEEE Trans. Instrum. Meas., vol. 59, no. 3, pp. 575-585, Mar. 2010.

[10] A. R. J. Ruiz, F. S. Granja, J. C. P. Honorato, and J. I. G. Rosas, "Accurate pedestrian indoor navigation by tightly coupling foot-mounted IMU and RFID measurements," IEEE Trans. Instrum. Meas., vol. 61, no. 1, pp. 178-189, Jan. 2012.

[11] M. Jiang, H. Shang, Z. Wang, H. Li, and Y. Wang, "A method to deal with installation errors of wearable accelerometers for human activity recognition," Physiol. Meas., vol. 32, no. 3, pp. 347-358, Mar. 2011.

[12] I. Skog, P. Händel, J.-O. Nilsson, and J. Rantakokko, "Zero-velocity detection-An algorithm evaluation," IEEE Trans. Biomed. Eng., vol. 57, no. 11, pp. 2657-2666, Nov. 2010.

[13] Z. Wang, H. Zhao, S. Qiu, and Q. Gao, "Stance-phase detection for ZUPT-aided foot-mounted pedestrian navigation system," IEEE/ASME Trans. Mechatronics, vol. 20, no. 16, pp. 3170-3181, Dec. 2015.

[14] L. Fang et al., "Design of a wireless assisted pedestrian dead reckoning system-The NavMote experience," IEEE Trans. Instrum. Meas., vol. 54, no. 6, pp. 2342-2358, Dec. 2005.

[15] A. M. Sabatini, "Quaternion-based extended Kalman filter for determining orientation by inertial and magnetic sensing," IEEE Trans. Biomed. Eng., vol. 53, no. 7, pp. 1346-1356, Jul. 2006.

[16] R. Mahony, T. Hamel, and J.-M. Pflimlin, "Nonlinear complementary filters on the special orthogonal group," IEEE Trans. Autom. Control, vol. 53, no. 5, pp. 1203-1218, Jun. 2008.

[17] M. S. Arulampalam, S. Maskell, N. Gordon, and T. Clapp, "A tutorial on particle filters for online nonlinear/non-Gaussian Bayesian tracking," IEEE Trans. Signal Process., vol. 50, no. 2, pp. 174-188, Feb. 2002.

[18] S. O. H. Madgwick, A. J. L. Harrison, and A. Vaidyanathan, "Estimation of IMU and MARG orientation using a gradient descent algorithm," in Proc. IEEE Int. Conf. Rehabil. Robot., Jan. 2011, pp. 1-7.

[19] D. Roetenberg, P. J. Slycke, and P. H. Veltink, "Ambulatory position and orientation tracking fusing magnetic and inertial sensing," IEEE Trans. Biomed. Eng., vol. 54, no. 5, pp. 883-890, May 2007.

[20] I. P. I. Pappas, M. R. Popovic, T. Keller, V. Dietz, and M. Morari, "A reliable gait phase detection system," IEEE Trans. Neural Syst. Rehabil. Eng., vol. 9, no. 2, pp. 113-125, Jun. 2001.

[21] E. Foxlin, "Pedestrian tracking with shoe-mounted inertial sensors," IEEE Comput. Graph. Appl., vol. 25, no. 6, pp. 38-46, Nov./Dec. 2005.

[22] Z.-Q. Zhang, W.-C. Wong, and J.-K. Wu, "Ubiquitous human upper-limb motion estimation using wearable sensors," IEEE Trans. Inf. Technol. Biomed., vol. 15, no. 4, pp. 513-521, Jul. 2011.

[23] S. Godha and G. Lachapelle, "Foot mounted inertial system for pedestrian navigation," Meas. Sci. Technol., vol. 19, no. 7, pp. 391-407, May 2008.

[24] A. Mannini and A. M. Sabatini, "Gait phase detection and discrimination between walking-jogging activities using hidden Markov models applied to foot motion data from a gyroscope," Gait Posture, vol. 36, no. 4, pp. 657-661, 2012.

[25] H. Fourati, "Heterogeneous data fusion algorithm for pedestrian navigation via foot-mounted inertial measurement unit and complementary filter," IEEE Trans. Instrum. Meas., vol. 64, no. 1, pp. 221-229, Jan. 2015.

[26] B. M. Biccard, "Relationship between the inability to climb two flights of stairs and outcome after major non-cardiac surgery: Implications for the pre-operative assessment of functional capacity," Anaesthesia, vol. 60 , no. 1, pp. 588-593, 2005.
[27] Y. Tao and H. Hu, "A novel sensing and data fusion system for 3-D arm motion tracking in telerehabilitation," IEEE Trans. Instrum. Meas. vol. 57, no. 5, pp. 1029-1040, May 2008.

[28] J. A. Hesch, D. G. Kottas, S. L. Bowman, and S. I. Roumeliotis, "Camera-IMU-based localization: Observability analysis and consistency improvement," Int. J. Robot. Res., vol. 33, no. 1, pp. 182-201, Jan. 2014.

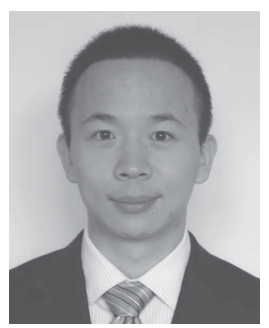

Sen Qiu received the B.S. degree in automation from the Dalian University of Technology, Dalian, China, in 2009, where he is currently pursuing the Ph.D. degree in control theory and control engineering with the School of Control Science and Engineering.

He had been a Visiting Researcher with the Department of Computer Science and Electronic Engineering, University of Essex, Colchester, U.K., from 2013 to 2014. His current research interests include gait analysis, body sensor network, and

pattern recognition.

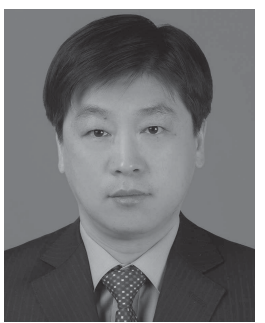

Zhelong Wang (M'04) received the B.Sc. and M.Sc. degrees in automatic control from the Dalian University of Technology, Dalian, China, in 1996 and 1999, respectively, and the Ph.D. degree in robotics from the University of Durham, Durham, U.K., in 2003.

He joined the School of Electronic and Information Engineering, Dalian University of Technology, in 2004, where he is currently a Professor. His current research interests include robotics, intelligent control, mechatronics, body sensor networks, and machine learning.

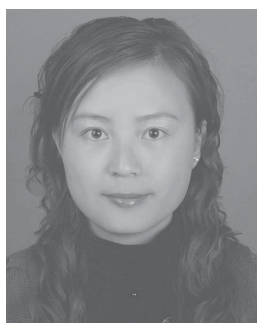

Hongyu Zhao received the B.S. degree in automation from the Dalian University of Technology, Dalian, China, in 2007, where she is currently pursuing the Ph.D. degree in control theory and control engineering with the School of Control Science and Engineering.

Her current research interests include pedestrian navigation, indoor localization, inertial navigation and positioning, and gait analysis.

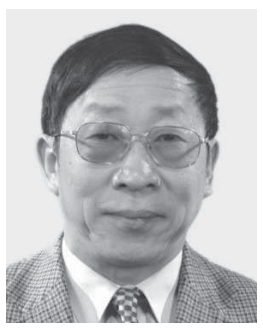

Huosheng Hu (M'94-SM'01) received the M.Sc. degree in industrial automation from Central South University, Changsha, China, in 1982, and the Ph.D. degree in robotics from the University of Oxford, Oxford, U.K., in 1993.

He is currently a Professor with the School of Computer Science and Electronic Engineering, University of Essex, Colchester, U.K., leading the robotics research. He has authored over 450 papers in the areas of robotics, human-robot interaction, and pervasive computing.

Dr. $\mathrm{Hu}$ is a Founding Member of the IEEE Robotics and Automation Society on Networked Robots, and a fellow of the Institution of Engineering and Technology and Institute of Measurement and Control. He has been the Program Chair and a member of the Advisory or Organizing Committee for many international conferences. He currently serves as an Editor-in-Chief of the International Journal of Automation and Computing, Digital Communications and Networks, and the Journal of Robotics, and an Executive Editor of the International Journal of Mechatronics and Automation. 